\title{
RECONSTRUCTION OF MEDIAL PATELLOFEMORAL LIGAMENT THROUGH TWO TRANSVERSE PATELLAR TUNNEL TECHNIQUE USING HAMSTRING AUTOGRAFT
}

\author{
Ajay Kumar 1 , Niloy Samanta², Subhrajyoti Sil ${ }^{3}$
}

${ }^{1}$ Associate Professor, Department of Orthopaedics, College of Medicine, Kalyani.

${ }^{2}$ Senior Resident, Department of Orthopaedics, RG Kar Medical College, Kolkata.

${ }^{3}$ Senior Resident, Department of Orthopaedics, College of Medicine, Kalyani.

ABSTRACT
BACKGROUND
Recurrent patellar dislocation affects mainly young active population. Medial patellofemoral ligament (MPFL), a condensation of
the medial retinaculum, is the main soft tissue restraint against lateral patellar displacement. There are many procedures to treat
patellar instability due to soft tissue reasons.

\section{MATERIALS AND METHODS}

We report the 24 months followup clinical results of 48 medial patellofemoral ligament reconstructions with semitendinosus tendon autograft using two transverse patellar tunnel technique in 48 patients having chronic patellar instability with no bony cause of recurrent patellar dislocation like patella alta or severe trochlear dysplasia.

\section{RESULTS}

At the end of 24 months followup, the average Kujala score was 93 (range: 80 - 100). No patellar re-dislocations or patella fracture were observed; 6 patients required manipulation under anaesthesia due to stiffness, but subsequently regained a satisfactory range of motion of knee.

\section{CONCLUSION}

This operative procedure provided good post-operative stability of patella and patient's satisfaction with no complications of postoperative patellar fracture.

\section{KEYWORDS}

Medial Patellofemoral Ligament, Patellar Dislocation, Hamstring Graft, Kujala Score, Patellofemoral Instability.

HOW TO CITE THIS ARTICLE: Kumar A, Samanta N, Sil S. Reconstruction of medial patellofemoral ligament through two transverse patellar tunnel technique using hamstring autograft. J. Evolution Med. Dent. Sci. 2017;6(87):6034-6039, DOI: $10.14260 /$ jemds $/ 2017 / 1312$

\section{BACKGROUND}

Recurrent patellar instability is a clinical problem affecting young, active population. Medial patellofemoral ligament (MPFL), a condensation of the medial retinaculum, is primary medial restraining structure to lateral displacement of the patella. MPFL tear occur in $94 \%$ of patients following a patellar dislocation. Healing capacity of MPFL is limited, which results in increased laxity of the medial patellar retinacular structures. ${ }^{[1,2]}$ There are many procedures to treat patellar instability with varying clinical results.[3,4] MPFL reconstruction is a popular method in the treatment of chronic patellar instability due to soft tissue reasons. We report the 24 months followup clinical results of 48 medial patellofemoral ligament reconstructions with semitendinosus tendon autograft and fixed through two transverse patellar tunnels with medial femoral condyle by bioabsorbable interference screw. We hypothesise that results of our procedure are comparable to previously published results in different studies of MPFL reconstruction and in addition the

'Financial or Other Competing Interest': None.

Submission 25-09-2017, Peer Review 18-10-2017,

Acceptance 25-10-2017, Published 30-10-2017.

Corresponding Author:

Dr. Ajay Kumar,

Associate Professor, Department of Orthopaedics,

College of Medicine, Kalyani, Nadia-741235.

E-mail: naveen_uno1@yahoo.co.in

DOI: $10.14260 /$ jemds $/ 2017 / 1312$ transverse placement of reconstructed MPFL through patella is more anatomical than longitudinal drill hole.

\section{MATERIALS AND METHODS}

In our study, we included 48 patients attending our institution with recurrent patellar instability. The patient's median age was 20 years (range: 17 - 24 years). There were 18 male and 30 female patients. All patients had chronic patellar instability with a minimum four total patellar dislocations; 18 patients had generalised ligament laxity according to Beighton score and none had severe grade of patella alta or trochlear dysplasia.

\section{Study Design \\ Case Series.}

\section{Inclusion Criteria \\ Patellar dislocation with-}

1. History of more than three episodes.

2. Low-grade/ no trochlear dysplasia.

3. Mild/ no patella alta.

\section{Exclusion Criteria}

1. Patients less than 16 years of age.

2. Acute dislocations.

3. Significant patellofemoral degenerative changes (grade III - IV). 
4. Significant patellar malalignment with an increased Q angle.

5. Tibial tubercle to trochlear groove distance (TTTG), greater than $15 \mathrm{~mm}$.

6. Severe trochlear dysplasia (Dejour type B or C).

7. High-grade patella alta (> 1.35 Caton-Deschamps index).

8. Any revision procedures or patients who had undergone previous knee surgery, except diagnostic arthroscopy.

9. Dislocations associated with other ligament injuries of knee.

Preoperative clinical evaluation was done to detect dynamic patellar tracking, patellofemoral osteoarthritis and to assess patellofemoral function by subjective Kujala score. Routine $x$-ray of the knee- antero-posterior, lateral and skyline views were done before the operation and at followup period.

Preoperative CT scan of knee was done for measuring TT$\mathrm{TG}^{[5]}$ distance. TT-TG is the quantitative indicator of $\mathrm{Q}$-angle. Caton-Deschamps index was used to evaluate patella alta.[6]

At followup, a subjective Kujala score was obtained. Clinical data included incidence of recurrent subluxation or dislocations. Any complications and re-operations were noted. The mean followup was 24 months (range: 20 26 months).

\section{Procedure}

The patient was placed in supine position. After administration of spinal anaesthesia, an above knee tourniquet was applied following the administration of prophylactic antibiotics. Skin preparation and sterile draping was performed in a standard manner. Semitendinosus tendon was harvested using a tendon stripper in the usual fashion. The tendon was debrided off muscle tissue and prepared with non-absorbable locking suture on both ends and preserved within an antibiotic soaked moist swab.

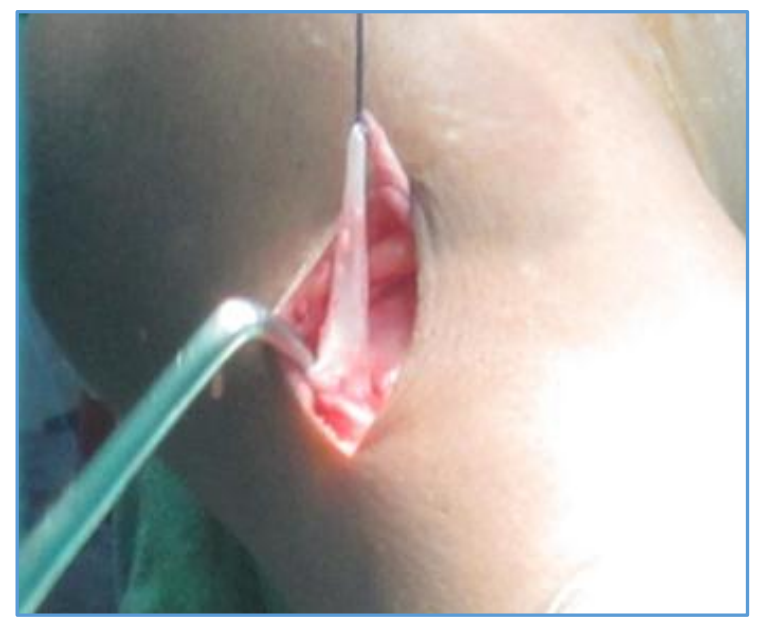

Figure 1. Showing Semitendinosus Graft

A midline incision was made on anterior aspect of patella. The pre-patellar fascia was elevated to expose the medial and lateral border of the patella. After stabilising the inferior portion of the patella, two beath pin were placed transversely, almost parallel to each other, $1 \mathrm{~cm}$ apart in the upper third of the patella. Two transverse tunnels were made along the beath pin using $4.5 \mathrm{~mm}$ sized drill bit to accommodate approximately $4 \mathrm{~mm}$ thickness of the graft.
Care was taken so that these drill holes should not violate chondral surface and anterior cortex of patella.

After localising the medial epicondyle of distal femur by palpation, one beath pin was introduced at the medial femoral condyle in between epicondyle and adductor tubercle along the transepicondylar axis towards the lateral side of femur. A medial blind tunnel of about $4 \mathrm{~cm}$ long was drilled along the beath pin to accommodate graft to an adequate depth to allow optimal graft tension. The graft was introduced through the transverse tunnels from medial to lateral and then from lateral to medial using the beath pin, so that the graft forms a loop through the patella. An incision was made over the medial edge of the patella. Dissecting scissors was used to make a tunnel between the $2^{\text {nd }}$ and $3^{\text {rd }}$ fascial layers of medial side of the knee aiming toward the medial epicondyle.

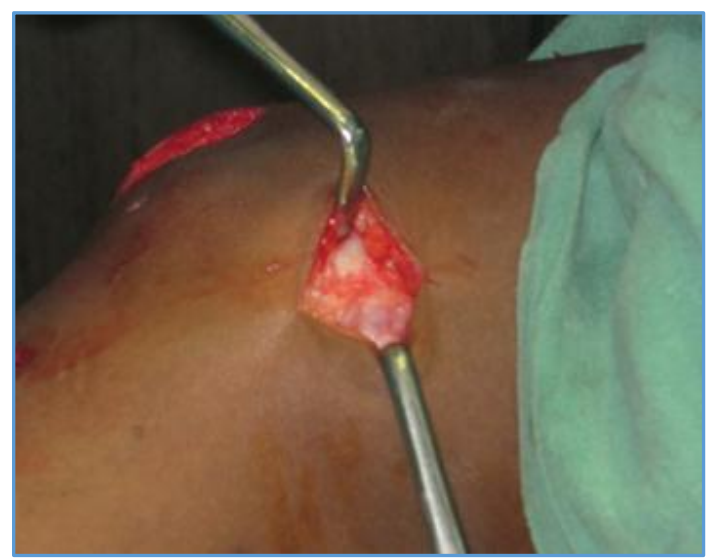

Figure 2. Showing Incision over Medial Epicondyle of Femur

The graft was passed through the plane between these layers. Then non-absorbable suture was passed along the transepicondylar axis using the Beath pin, pulling the graft into the medial tunnel and the patella was positioned in the femoral trochlea keeping the graft under tension on lateral side, knee was cycled several times from full flexion to full extension to pre-stretch the graft. Both ends of the graft were then secured within the medial epicondyle tunnel using a bioabsorbable interference screw keeping the knee in between $20^{\circ}$ and $60^{\circ}$ of flexion, depending on where the patella was engaging in the trochlear groove while applying low tension pull over the graft $(2 n)$. Retinacula were sutured back to the patella and then closure of subcutaneous tissues and skin were done. Long leg slab in knee extension were applied following routine dressings and bandages.

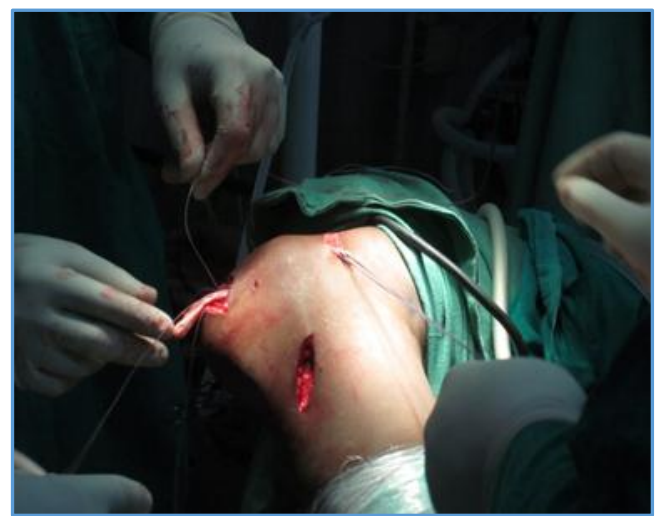

Figure 3. Showing Passage of Graft through $2^{\text {nd }}$ Layer on Medial Side of Knee 


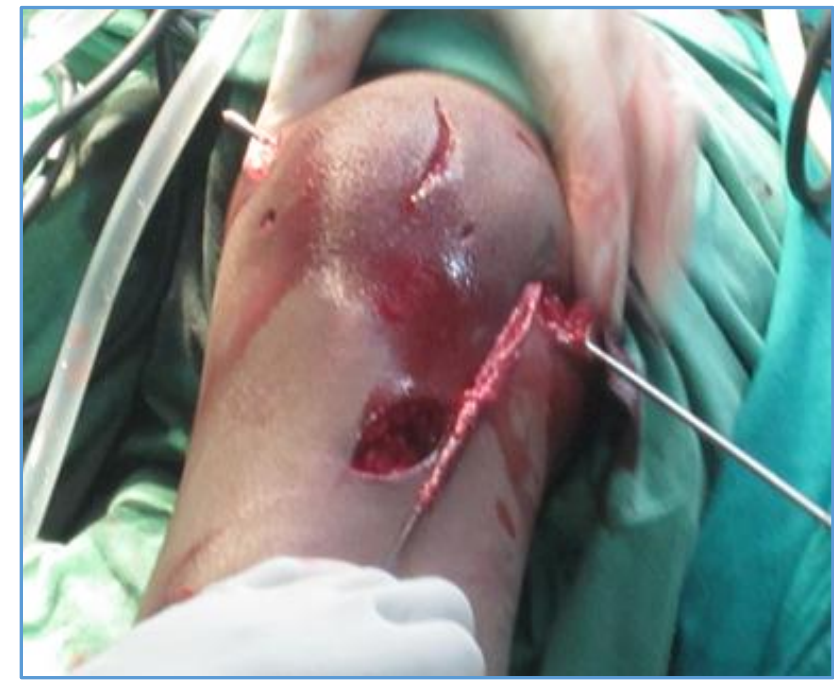

Figure 4. Showing Position of Beath Pin along Transepicondylar Axis

\section{Postoperative Rehabilitation}

After 2 weeks of operation, sutures and slab were removed and long knee brace were applied to immobilise knee in extension. Patients were allowed partial weight bearing as tolerated with support of axillary crutches keeping the knee in extension in brace for next 2 weeks. During this period brace was unlocked 3 - 4 times in a day and active range of motion of knee 0 - 90 degrees and light isometric quadriceps exercises were allowed. After 4 weeks, full weight bearing and intensive quadriceps rehabilitation programs were started. Controlled sports were allowed after three months and contact sports activities were permitted after six months.

\section{RESULTS}

The clinical results of 24 months followup in 48 patients who had undergone MPFL reconstruction were satisfactory. No redislocation of patella had occurred; 12 patients had tenderness on femoral screw fixation site on deep palpation. Manipulation under anaesthesia was required for 6 patients because their knee flexion was less than $90^{\circ}$, but subsequently satisfactory range of motion was restored. No post-operative patellar fracture occurred. The results of the passive patella glide test indicated more stable patella. The average preoperative Kujala functional score was 42.8 and at the end of 2 years after operation average Kujala score was 93 points (range: 80 - 100 points).

\section{DISCUSSION}

Stability of patellofemoral joint depends on- (A) Soft tissue like quadriceps muscle, specially vastus medialis obliquus, medial retinaculum, medial patellofemoral ligament (MPFL), medial patellotibial ligament and lateral retinaculum[7]; (B) Bony factors like primarily patellar shape, patellar tilt, tibial tubercle-trochlear groove distance $(\mathrm{Q}$-angle quantification by CT scan), patella alta and trochlear dysplasia.[8] Secondary bony instability factors are excessive external femoral or tibial rotation, genu recurvatum and genu valgum. These instability factors predispose to disruption of the MPFL, knee joint capsule, medial patellar retinaculum and/or vastus medialis obliquus leads to recurrent patellar instability.
Major constraints of patella during early knee flexion $\left(<30^{\circ}\right.$ flexion) are the soft tissue structures, but after $30^{\circ}$ patellar stability depends on mainly bony structures specially trochlea of distal femur.[9]

MPFL is a distinct condensation of medial capsular fibres of knee joint in coronal plane present as distinct soft tissue structure within the medial retinaculum, which originates from the posterior aspect of medial epicondyle, adductor tubercle and adductor tendon as well as the MCL. MPFL runs transversely deep into the distal vastus medialis obliquus and inserts at the superior two-thirds of medial border of patella. MPFL lies in the middle or second layer of medial side of knee joint where parapatellar retinaculum and superficial MCL are also present. It is approximately $55 \mathrm{~mm}$ long and its width ranges from 5 to $12 \mathrm{~mm} .{ }^{[10]} \mathrm{In}$ full extension with the quadriceps contracted MPFL is most taut and assists in guiding the patella into the trochlea during the early stages of flexion ( 0 - 30 degrees). Vastus medialis tendon and the MPFL have common attachment to the superomedial patella, so they act as a dynamic element for stability of patellofemoral joint. Biomechanical studies have shown that the MPFL is the major soft tissue restraint contributing an average $53 \%$ of the total force that prevents lateral displacement of patella.[11] Desio found similar values of $60 \%$ at $20^{\circ}$ knee flexion.[12] This ligament has a mean tensile strength of 208 n.[13]

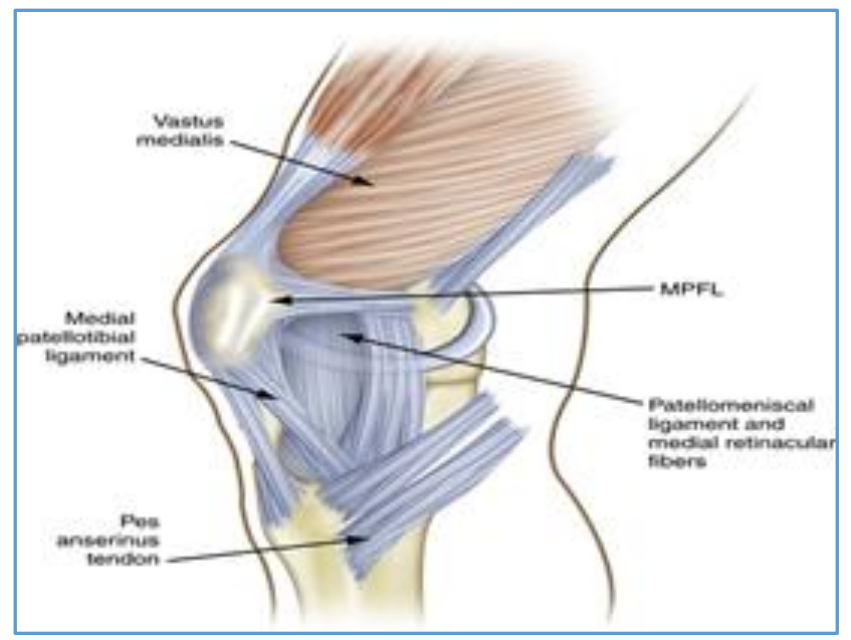

Figure 5. Showing Anatomical Position of MPFL

Patellofemoral dislocations tend to occur as a result of quadriceps contraction across a flexed, valgus knee with the weight bearing tibia externally rotated relative to the femur.[14] MPFL tear occur in $94 \%$ of patients following a patellar dislocation. In $70 \%$ cases tear occur at the patellar insertion site, while in almost all of remaining cases damage seen at the femoral origin. In very rare cases, there is also interstitial damage.[15]

MPFL disruption clinically may present with recurrent patellar instability to lateral direction, with or without pain and tenderness along the medial retinaculum. Radiography of bilateral knee joints (AP, lateral, skyline view) and CT scan help to rule out any insulting bony factor, which predispose to patellar dislocation. MRI confirms the MPFL disruption. 


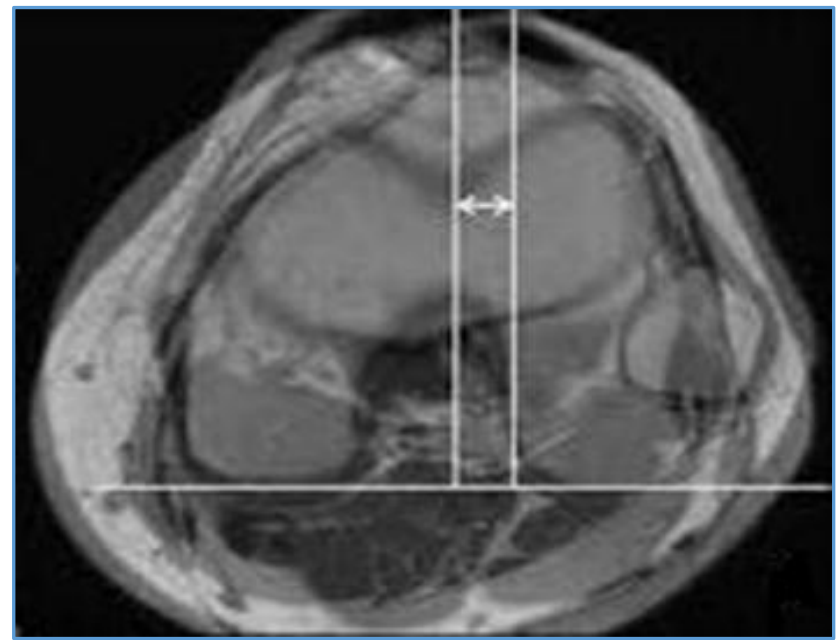

Figure 6. Showing TT-TG Distance in Overlapped Image of MRI

Conservative management is a recommended option following first episode of patellar dislocation.[16][17] Conservative management includes exercises to strengthen the quadriceps, especially the vastus medialis to prevent further instability. If the dislocation recurs, then operative intervention is considered with the aim of restoring the soft tissue anatomy to normal as much possible with or without bony procedure.

The optimal surgical treatment for chronic patellar instability remains controversial. The reconstructive techniques have an aim to restore this medial tether of the patella. Various tendon sources have been described including gracilis, semitendinosus, partial patellar tendon and allografts or artificial tendons. Grafts can be fixed using a variety of techniques including patellar drill holes, interference screws, sutures and suture anchors at the medial femoral condyle. Proximal or distal realignment procedures can be considered as treatment for recurrent patellar dislocation, but associated knee osteoarthritis in these nonanatomical surgeries leads to poor results.[18][19][20][21] Medial transfer of the tibial tubercle increases joint loading within the medial tibiofemoral compartment and the medial facet of the patellofemoral joint and induces variable changes within the lateral tibiofemoral compartment.[18]

MPFL reconstruction gives better results in recurrent patellar dislocations than with non-anatomical reconstruction, which may alter the biomechanics of the patellofemoral joint.[22][23][24][25] The association of definite knee osteoarthritis in MPFL reconstruction with or without lateral release was small in the long-term followup.[19][20][21][25]

In cadaver study, MPFL reconstruction showed a significant reduction in lateral displacement of patella and joint load compared with medial transfer of the tibial tuberosity. Hence, medial patellofemoral ligament reconstruction is an anatomical reconstruction. Now, it is generally accepted that reconstruction of the MPFL is a good procedure to correct chronic patellar instability, particularly in patients who have history of recurrent patellar dislocations associated with generalised ligamentous hyperlaxity and for patients who have obligatory patellar dislocations. Medial retinacular tissue is incompetent in both of these conditions.[26] In absence of intact MPFL, patellar tracking is significantly affected by a laterally directed force, but returns to normal following reconstruction of MPFL.[27]

Patient's selection is very much important while considering for MPFL reconstruction. Before doing MPFL reconstruction, it is important to distinguish common patellofemoral pain syndrome from anterior pain associated with patellar instability. Some studies have shown poorer outcomes in patients with articular cartilage damage at the time of surgery.[28][29] In case of advanced patellar chondral damage (grade III - IV), a patellofemoral offloading procedure such as a Fulkerson's osteotomy is more appropriate than MPFL reconstruction alone. So these patients were not included in our study. Patients with a markedly increased TTTG distance would benefit from bony surgical procedures and not only by MPFL reconstruction. So in our study, we excluded the patients having TT-TG distance more than 15 mm. Significant trochlear dysplasias like Dejour type B and Dejour type $C$ are contraindications to MPFL reconstruction in isolation. Trochleoplasty possibly in combination with MPFL reconstruction are needed in these patients. One study[30] did not find poorer results in patients with mild trochlear dysplasia who underwent only MPFL reconstruction. So in our study, patients having Dejour type A trochlear dysplasia were included.

Different methods are there to reconstruct the MPFL with hamstring graft and variation are also present between patellar tunnel placement and graft fixation methods. Many patients with patellar instability may have generalised ligamentous hyperlaxity or trochlear dysplasia.[31] So reconstruction of MPFL is done with stronger tissue than original tissue to compensate for the underlying predisposing pathology without changing the original position of the patella and its original conformity with its underlying trochlea.[32][33] Semitendinosus and gracilis tendon are commonly harvested as grafts for reconstructive procedures.[34] But we used semitendinosus tendon as the gracilis tendon is very thin. If stiff graft is used for MPFL reconstruction, then patellofemoral joint loading can be largely increased if small errors occur in graft length and/or attachment site.[35]

More proximally placed graft will result in a reconstructed MPFL, that is lax in extension and tight in flexion that might cause loss of knee flexion and creates excessive pressure on the medial patella.[35] Conversely, distally placed graft will result in an overtight MPFL in extension and a lax ligament in flexion. To optimise graft tension, we did cycling the knee through its range of motion prior to fixation. This aims to remove "give" from the graft prior to fixation. Over tensioning could be avoided by applying low loads (2n) to reconstructed MPFL graft during fixation to medial femoral condyle, so that normal translation and patellofemoral contact is re-established.[36] It has been shown in many anatomical studies that graft is to be $5-12 \mathrm{~mm}$ wide. Single strand techniques use hamstrings of about 3.5 - $4 \mathrm{~mm}$ wide. This can be doubled to give a thicker graft, but may cause difficulty with the placement of a larger single tunnel within the patella. A larger patellar tunnel may increase the risk of joint penetration or patellar fracture. Our two tunnel technique allows a wider tendon graft, but utilises small tunnels diameter, thus minimising those potential complications compared to a single tunnel 
technique. As the tunnels traverse the entire width of the patella, this technique may also influence patellar tilt. Further transverse placement of graft through patella is more anatomical than longitudinal patellar drill-hole technique. Schöttle et al reported that the mean Kujala score improved significantly from 55.0 to 85.7 points in his study. Reconstruction of the MPFL was done with gracilis tendon autograft in transverse patellar drill holes by Christiansen et al. In their study, knee function score improved overall from 46 points (range 12 - 67 points) to 84 points (range $62-100$ points) at followup. In Mikashima et al study, the mean Kujala score was 95.2 postoperatively. These results are comparable with our outcome.

\section{CONCLUSION}

This transverse double tunnel technique allows more anatomical placement of wider reconstructed ligament, minimising graft impingement with good post-operative patellar stability without increasing the risks of patellar fracture compared to a single large tunnel technique. Subjective outcome scores is compatible with other reported techniques. Patient's selection remains vitally important to ensure optimal surgical outcomes.

\section{REFERENCES}

[1] Sallay PI, Poggi J, Speer KP, et al. Acute dislocation of the patella. A correlative pathoanatomic study. Am J Sports Med 1996;24(1):52-60.

[2] Nomura E. Classification of lesions of the medial patello-femoral ligament in patellar dislocation. Int Orthop 1999;23(5):260-3.

[3] Davis DK, Fithian DC. Techniques of medial retinacular repair and reconstruction. Clin Orthop Relat Res 2002;402:38-52.

[4] Hughston JC. Subluxation of the patella. J Bone Joint Surg Am 1968;50(5):1003-26.

[5] Goutallier D, Bernageau J, Leucudonnec B. The measurement of the tibial tuberosity. Patella groove distanced technique and results. Rev Chir Orthop Reparatrice Appar Mot 1978;64(5):423-8.

[6] Caton J, Deschamps G, Chambat P, et al. Patella infera. Apropos of 128 cases. Rev Chir Orthop Reparatrice Appar Mot 1982;68(5):317-25.

[7] Fulkerson JP. Evaluation of the peripatellar soft tissues and retinaculum in patients with patellofemoral pain. Clin Sports Med 1989;8(2):197-202.

[8] Dejour H, Walch G, Nove-Josserand L, et al. Factors of patellar instability: an anatomic radiographic study. Knee Surg Sports Traumatol Arthrosc 1994;2(1):1926.

[9] Heegaard J, Leyvraz PF, van Kampen A, et al. Influence of soft tissue structures on patellar three dimensional tracking. Clin Orthopaedics Relat Res 1994;299:23543.

[10] Reider B, Marshall JL, Koslin B, et al. The anterior aspect of the knee joint. J Bone Joint Surg Am 1981;63(3):351-6.

[11] Conlan T, Garth WP, Lemons JE. Evaluation of the medial soft-tissue restraints of the extensor mechanism of the knee. J Bone Joint Surg 1993;75(5): 682-93.
[12] Desio SM, Burks RT, Bachus KN. Soft tissue restraints to the lateral patellar translation in the human knee. Am J Sports Med 1998;26(1):59-65.

[13] Mountney J, Senavongse W, Amis AA, et al. Tensile strength of the medial patellofemoral ligament before and after repair or reconstruction. J Bone Joint Surg Br 2005;87(1):36-40.

[14] Boden BP, Pearsall AW, Garrett WE, et al. Patellofemoral instability: evaluation and management. J Am Acad Orthop Surg 1997;5(1):47-57.

[15] Garth WP, DiChristina DG, Holt G. Delayed proximal repair and distal realignment after patellar dislocation. Clin Orthop Relat Res 2000;377:132-44.

[16] Nikku R, Nietosvaara Y, Aalto K, et al. Operative treatment of primary patellar dislocation does not improve medium-term outcome: a 7-year follow-up report and risk analysis of 127 randomized patients. Acta Orthop 2005;76(5):699-704.

[17] Buchner M, Baudendistel B, Sabo D, et al. Acute traumatic primary patellar dislocation: long term results comparing conservative and surgical treatment. Clin J Sport Med 2005;15(2):62-6.

[18] Huberti HH, Hayes WC. Patellofemoral contact pressures. The influence of q-angle and tendofemoral contact. J Bone Joint Surg Am 1984;66(5):715-24.

[19] Ahmed AM, Burke DL, Hyder A. Force analysis of the patellar mechanism. J Orthop Res 1987;5(1):69-85.

[20] Pidoriano AJ, Weinstein RN, Buuck DA, et al. Correlation of patellar articular lesions with results from anteromedial tibial tubercle transfer. Am J Sports Med 1997;25(4):533-7.

[21] Nomura E, Inoue M, Kobayashi S. Long-term follow-up and knee osteoarthritis change after medial patellofemoral ligament reconstruction for recurrent patellar dislocation. Am Sports Med 2007;35(11):1851-8.

[22] Mikashima Y, Kimura M, Kobayashi Y, et al. Clinical results of isolated reconstruction of the medial patellofemoral ligament for recurrent dislocation and subluxation of the patella. Acta Orthop Belg 2006;72(1):65-71.

[23] Ostermeier S, Stukenborg-Colsman C, Hurschler C, et al. In vitro investigation of the effect of medial patellofemoral ligament reconstruction and medial tibial tuberosity transfer on lateral patellar stability. Arthroscopy 2006;22(3):308-19.

[24] Smith TO, Walker J, Russell N. Outcomes of medial patellofemoral ligament reconstruction for patellar instability: a systematic review. Knee Surg Sports Traumatol Arthrosc 2007;15(11):1301-14.

[25] Panagopoulos A, van Niekerk L, Triantafillopoulos IK. MPFL reconstruction for recurrent patella dislocation: a new surgical technique and review of the literature. Int J Sports Med 2008;29(5):359-65.

[26] Andrish J. The management of recurrent patellar dislocation. Orthop Clin North Am 2008;39(3):313-27.

[27] Sandmeier RH, Burks RT, Bachus KN, et al. The effect of reconstruction of the patellofemoral ligament on patellar tracking. Am J Sports Med 2000;28(3):345-9. 
[28] Christiansen SE, Jacobsen BW, Lund B, et al. Reconstruction of the medial patellofemoral ligament with gracilis tendon autograft in transverse patellar drill holes. Arthroscopy 2008;24(1):82-7.

[29] Gomes EJL. Medial patellofemoral ligament reconstruction for recurrent dislocation of the patella: a preliminary report. Arthroscopy 1992;8(3):335-40.

[30] Schöttle PB, Fucentese SF, Romero J. Clinical and radiological outcome of medial patellofemoral ligament reconstruction with a semitendinosus autograft for patella instability. Knee Surg Sports Traumato Arthrosc 2005;13(7):516-21.

[31] Neyret P, Robinson AH, Le Coultre B, et al. Patellar tendon length-the factor in patellar instability? Knee 2002;9(1):3-6.

[32] Erasmus PJ. Reconstruction of the medial patellofemoral ligament in recurrent dislocation of the patella. Arthroscopy Supplement 1998;14:42.
[33] Hamner DL, Brown CH, Steiner ME, et al. Hamstring tendon grafts for reconstruction of the anterior cruciate ligament: biomechanical evaluation of the use of multiple strands and tensioning techniques. J Bone Joint Surg Am 1999;81(4):549-57.

[34] Mologne TS, Friedman MJ. Arthroscopic anterior cruciate ligament reconstruction with hamstring tendons: indications, surgical technique and complications and their treatment. In: Scott WN. edr. Surgery of the knee. $4^{\text {th }}$ edn. Philadelphia: Churchill Livingstone 2006;648-51.

[35] Elias JJ, Cosgarea AJ. Technical errors during medial patellofemoral ligament reconstruction could overload the medial patellofemoral cartilage: a computational analysis. Am J Sports Med 2006;34(9):1478-85.

[36] Beck P, Brown NA, Greis PE, et al. Patellofemoral contact pressures and lateral patellar translation after medial patellofemoral ligament reconstruction. Am J Sports Med 2007;35(9):1557-63. 